\title{
Experimental study of alternating magnetic field in laminated ferromagnetic core
}

\author{
Yuriy Adamyan ${ }^{1, *}$, Sergey Krivosheev ${ }^{1}$, and Sergey Magazinov ${ }^{1}$ \\ ${ }^{1}$ Peter the Great St.Petersburg Polytechnic University, Polytechnicheskaya 29, St. Petersburg, 195251, \\ Russian Federation
}

\begin{abstract}
At engineering simulations of magnetic fields in ferromagnetic cores standard Preisach and Jiles-Atherton models are commonly used. At that a material magnetic properties are considered similar in all points of a core. However, the view of experimental hysteresis loops can differ from calculated even for materials with well-known magnetic properties, e.g. transformer steel. Experimental registration of hysteresis loops in characteristic zones of laminated steel core have revealed substantial discrepancy as in the loops view as in the field strength distribution in comparison with calculation results. Measurements at the frequency and field amplitude variation in order to reveal their influence on the magnetization process are conducted. The results obtained point to possible significant influence of magnetostriction effect on functioning of the ferromagnetic core. This influence can appear at frequencies of the core mechanical resonance and lead to changes in the core inductance and quality factor. The effect is relatively weakly pronounced at laminated steel core, but in some cases needs to be considered, e.g. if big size core operates at network frequency.
\end{abstract}

\section{Introduction}

Engineering simulations of magnetic fields in ferromagnetics are of importance for magnetic cores $[1,2,3]$, technology (e.g. non-destructive testing $[3,4,5,6]$ ). Commonly, the magnetic properties are considered similar in all points of the core. Material models used for FEM computer simulations connect magnetic flux density and field strength at a point. The most standard are Preisach and Jiles-Atherton (JA) models [7]. In fact, the important factor influencing on the magnetic properties is mechanical stress [8], which can be generated by magnetic pressure or (and) magnetostriction. In real objects the view of experimental hysteresis loops can differ from calculated even for materials with wellknown magnetic properties [10], e.g. transformer or construction [11] steel. In the E-type core the effect of mechanical stress can be pronounced in core regions with local stress concentration. In presented work the local values of the components of the vectors B and $\mathrm{H}$ in the laminated core are measured. Also the integral parameters of the coil are analysed with respect of the core mechanical frequency and field strength.

\footnotetext{
* Corresponding author: wiradam@ rambler.ru
} 


\section{Methods}

\subsection{Experimental setup}

The standard method of local magnetic flux density components measurements in laminated ferromagnetic core is forming of orthogonal turns of wire passed through the holes in the material [12]. At field strength measurements in a ferromagnetic sheet, actually, the tangent component of the field strength in outside, close to the media boundary can be used taking into account the tangent component continuity. Thin, multi-turn probes close to the steel surface are used. For the accuracy improvement, a kind of screen can be used for equalizing the spatial distribution of vacuum field strength [13].

The laminated hot rolled transformer steel is chosen for low-frequency measurements. As for the $\mathrm{H}$ - probes layout, we use probes, placed in the gap between steel sheets. This technique is described in [14], where the field strength was measured in the gap between coaxial parts of the ferrite ring core. It is shown that the error, caused by the finite probe thickness, is not higher that $10-15 \%$. The steel sheets thickness limits the maximal value of the field frequency. The estimation based on the skin layer size shows that for the $0.5 \mathrm{~mm}$ steel sheet, maximal frequency is about $1 \mathrm{kHz}$. The core magnetization is provided by the winding placed on the core central bar (Fig. 1). The winding feeding circuit consists of audio generator and battery fed bridge power amplifier. The excitation winding is connected in parallel with capacitor, forming the resonant circuit. Measurements were conducted for the probes placed in the core characteristic points, namely: in the rod middle (zone I), close to the internal corner (II) and in the corner part center (III).

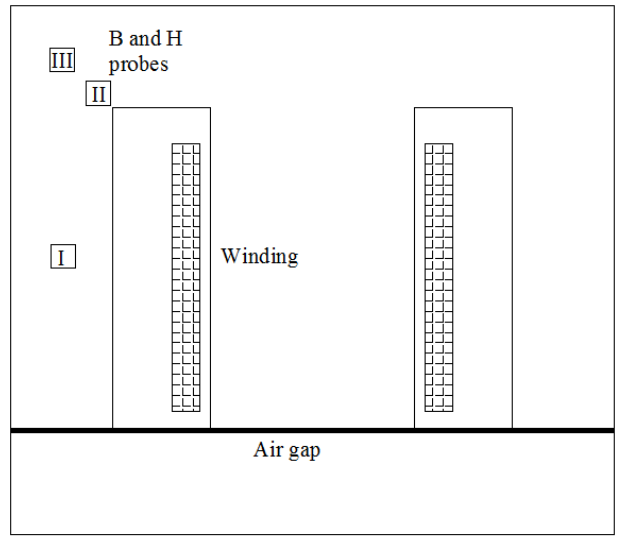

Fig. 1. Field probes layout.

\section{Results}

\subsection{The results of magnetic fields measurements in the core characteristic zones}

The working frequency $780 \mathrm{~Hz}$, corresponding to the electrical resonance in the circuit at capacity $80 \mu \mathrm{F}$, was used in the primary measurements series.

Preliminary calculations had shown that at this frequency the eddy currents influence on the field distribution inside the sheet is not substantial. Fig. 2a displays the experimental hysteresis loop in the core rod (zone I) and the loop obtained as the result of calculations 
using standard JA model, included into COMSOL 5.3 software package. It can be seen that the loops are close enough.
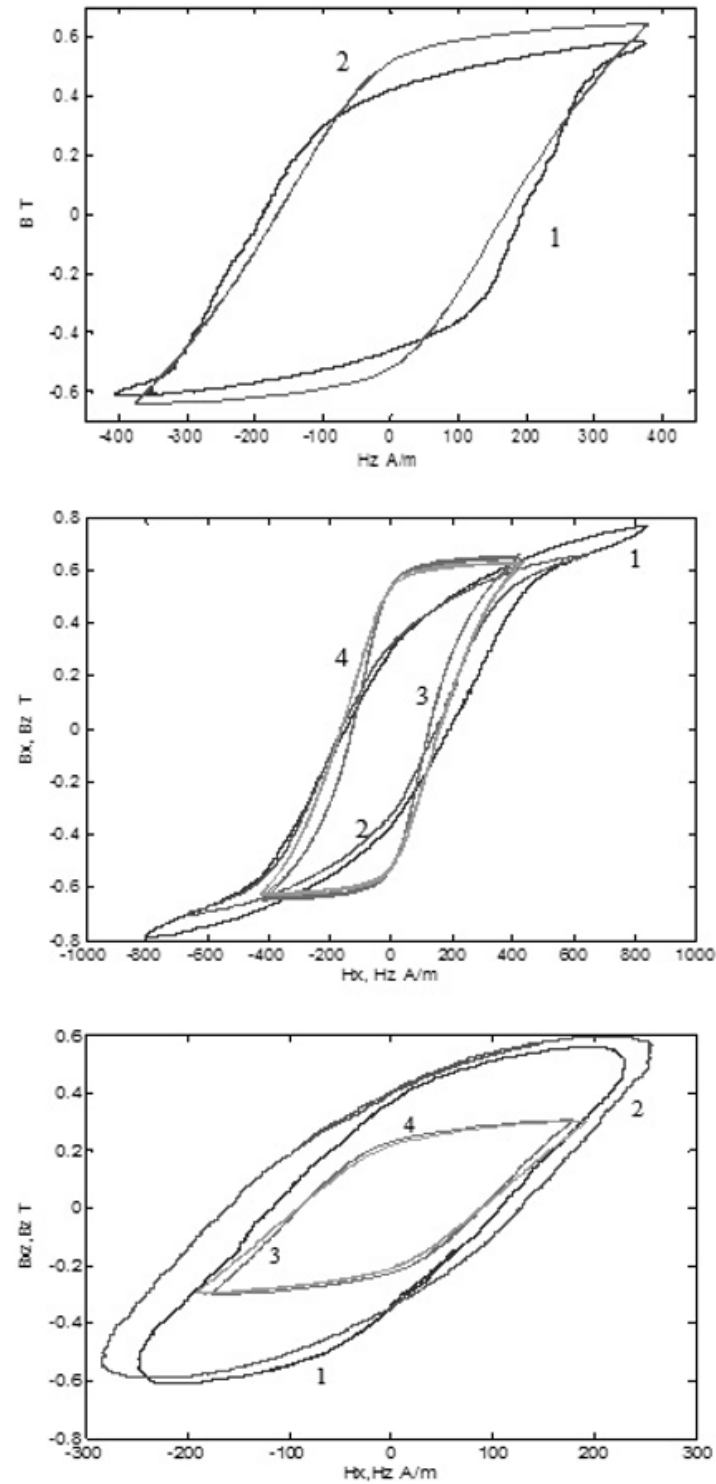

Fig. 2. Experimental and calculated hysteresis loops in the core characteristic points: a - zone I (1experiment, 2-calculation), b - zone II (1, 2-experiment, 3, 4-calculation), c - zone III (1, 2experiment, 3, 4-calculation).

At the same time, comparison of the experiment and calculation results at the corner zone demonstrates discrepancy as in the loops view as in the field strength (Fig. 2b and c). The characteristic "rounded" view of the shape of the field components loops at zone III indicates significant phase shift between $\mathrm{B}$ and $\mathrm{H}$.

These results has stimulated conducting of additional measurements in zone I (with practically uniform field structure) at the frequency and amplitude variation in order to reveal their influence on the magnetization process character. 


\subsection{Influence of the field frequency and amplitude on the core magnetization process}

\subsubsection{Influence of the excitation current frequency}

For the conducting of experiments on the frequency influence the constant excitation current amplitude mode is chosen. This amplitude is $30 \%$ smaller than in previous experiments. The resonant circuit capacity is taken as argument. At each capacity value, the generator output frequency is tuned to resonance at gradual rising of the excitation current up to fixed value (15 A at 14 turns in the winding). The frequency is varied from 0.5 to 1.5 $\mathrm{kHz}$. Besides of the resonant frequency, the magnetic field strength and coil (capacitor) voltage are registered. The equivalent inductance is determined from the capacity, resonant frequency and loss resistance.

The measurements results are displayed on Fig. 3. The data of electric measurements are juxtaposed with frequencies of the core mechanical resonances, obtained using wide band piezoelectric probe at the core mechanical oscillations excitation by periodic square shaped current pulses.
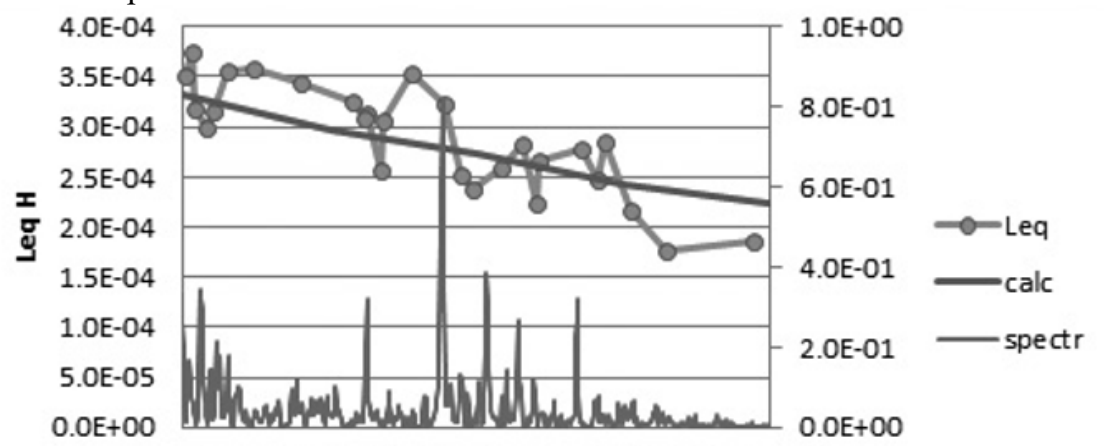

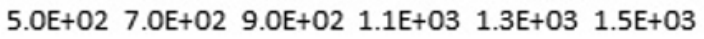

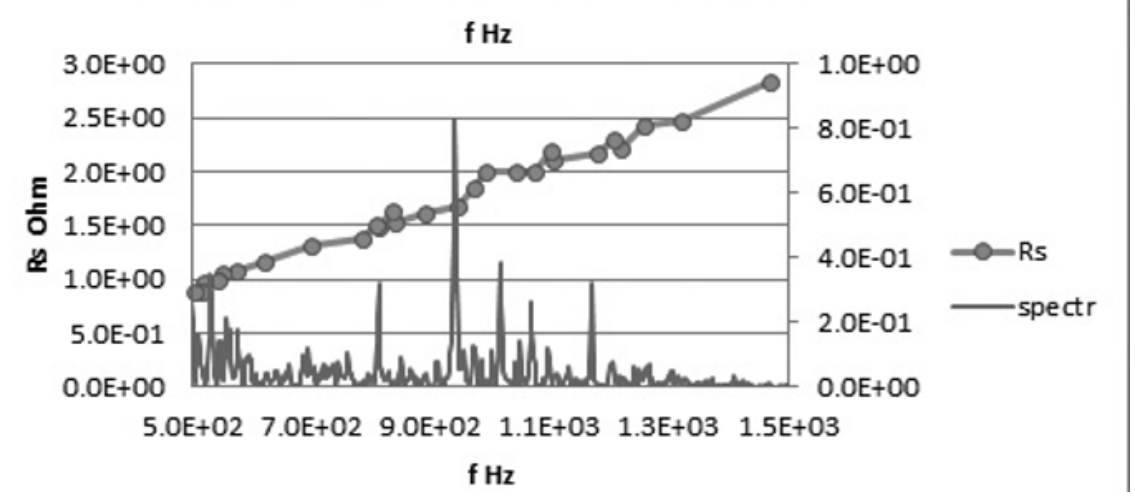




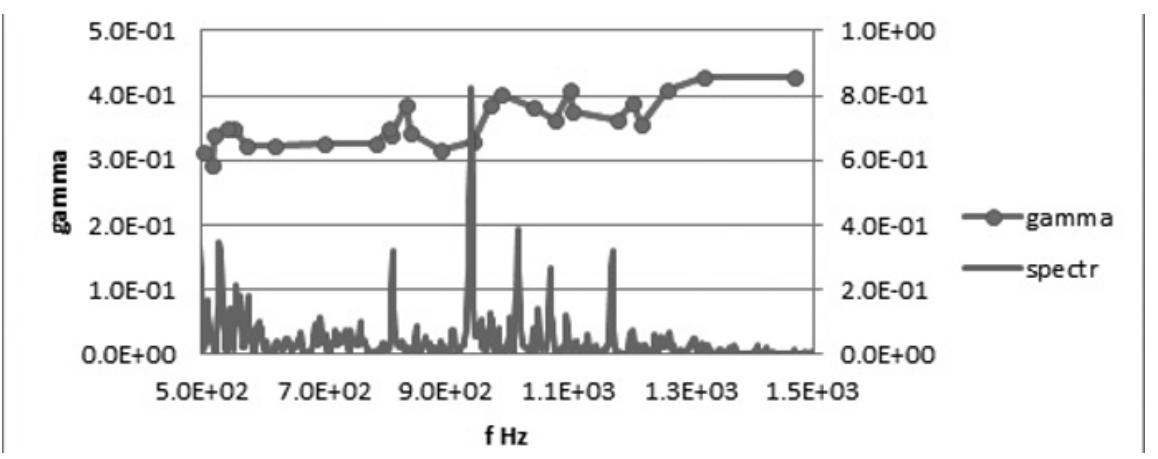

Fig. 3. Frequency dependences of the magnetic system inductance (a), loss resistance (b) and circuit parameter $\gamma(\mathrm{c})$.

As it follows from the data displayed, the sharp changes of electric parameters take place at frequencies, close to the some mechanical resonances. The inductance drop is accompanied with rise of the loss resistance and, respectively, reduction of the circuit quality factor. The circuit parameter

$$
\gamma=\frac{1}{2} R_{S} \sqrt{\frac{C}{L}}
$$

reverse to quality factor is presented on Fig. 3c. The most pronounced is this effect at the frequencies, close to the lines of the mechanical spectrum 528,812, 1016 and $1069 \mathrm{~Hz}$, where the quality factor drops by $2030 \%$. At the same time, the quality factor and inductance significantly rise at the vicinity of the most intensive spectrum line $940 \mathrm{~Hz}$. The "calc" curve on Fig. 3, corresponds to approximate calculation of the coil inductance with taking into account the magnetic field diffusion.

\subsubsection{Influence of the field amplitude}

The character of the hysteresis loop in the core bar visibly changes at the rise of the field amplitude. The hysteresis loops for core zone I, obtained at field amplitudes 0.4 (1) and 0.6 $\mathrm{T}(2)$ and frequency $780 \mathrm{~Hz}$ are presented on Fig. 4. As it can be seen from the oscillograms (Fig.5) of the field strength (1) and the magnetic flux density (2), change in the loops shape is related with sharp time shift of the $\mathrm{H}$ - curve maximum at the approach to some threshold of B value (slightly higher than $0.4 \mathrm{~T}$ ).

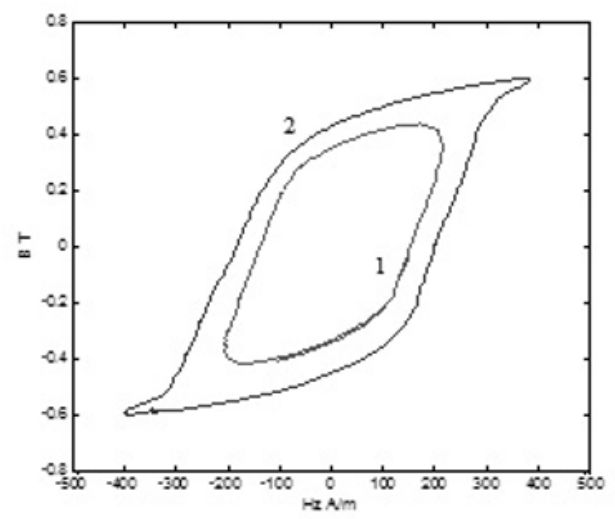

Fig. 4. Hysteresis loops obtained at magnetic flux densities 0.4 and $0.6 \mathrm{~T}$. 
At given capacity the resonant frequency substantially depends on the excitation current amplitude. It is well known that for most ferromagnetics a magnetic permeability dependence on field strength has maximum at low field. At frequency range free from pronounced mechanical resonances the dependence of electric resonant frequency vs current has corresponding minimum (Fig. 6a). If the mechanical resonant frequency, influencing on the electric parameters, presents in the frequency range analyzed, the dependence can be of anomalous character. It can be seen from Fig. $6 \mathrm{~b}$ that the curve has the bend, corresponding to the frequency $812 \mathrm{~Hz}$ of the mechanical resonance. Similarly dependence behaves at the frequency range around group of mechanical resonant lines 528 $550 \mathrm{~Hz}$ (Fig. 6c), though the anomaly is less pronounced.
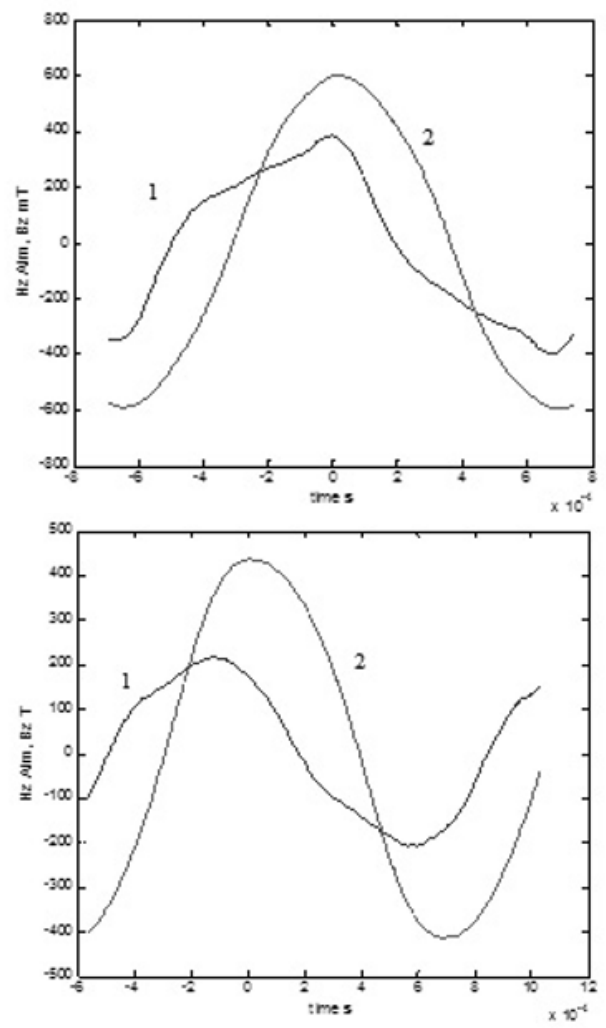

Fig. 5. Signals of $\mathrm{H}$ and $\mathrm{B}$ in the bar zone at magnetic flux densities 0.4 and $0.6 \mathrm{~T}$. 

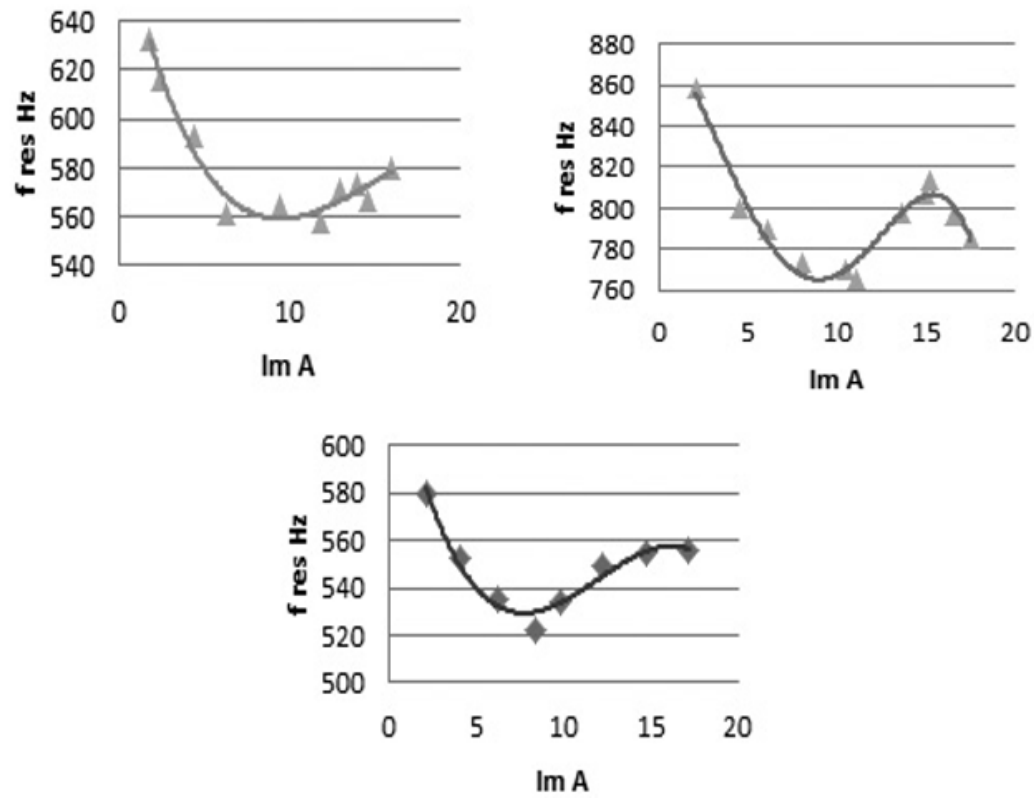

Fig. 6. Dependences of the electric resonant frequency vs current: a - $C=126.5 \mu \mathrm{F}, \mathrm{b}-\mathrm{C}=65 \mu \mathrm{F}, \mathrm{c}-$ $\mathrm{C}=135.5 \mu \mathrm{F}$.

\section{Discussion}

\subsection{Results summary}

At the experiment, hysteresis loops in different zones of the core has different character. In the zone I (core bar) the loops can be considered as close to standard form and quite well described by JA model. In contrary, in the corner zones (II and III) discrepancies between calculation and experiment are drastic. It can be supposed that the mechanical stress influences on the local magnetic properties of the material. Study of the circuit resonant frequency dependence on the current allows revealing the frequency domains where the deviations from standard dependence present at frequencies close to some core mechanical resonant frequencies. Revealed anomalies of as integral (inductance, loss resistance) as local (magnetic permeability, phase shift between $\mathrm{H}$ and $\mathrm{B}$ ) parameters depending on field strength and frequency, indicate their relation with mechanical resonant frequencies of the core.

The expected cause of the observed phenomena is the effect of magnetostriction. At mechanical resonant frequencies oscillations of the core can produce significant mechanical stresses and bending deformations in corner zones. It leads to generation of additional magnetic fields due to reverse magnetostriction effect $[15,16,17]$. Despite of relatively low magnetostriction constant of the transformer steel, local concentration of stress in the corner zones can result in deformation of hysteresis loops and significant change of the coil integral parameters. The absence of anomalies of the circuit resonant frequency dependence on the current at low fields can be explained considering that the reverse magnetostriction effect in steel at small mechanical stresses becomes maximal around field strength 300 $400 \mathrm{~A} / \mathrm{m}$ [18]. Possibly, it explains why this effect has not been observed in low field measurements [19]. 


\subsection{Conclusion}

It is known that magnetostriction effect can cause vibration, noise leading to mechanical troubles in power equipment $[20,21,22]$ The presented results point to possible significant influence of magnetostriction effect on electromagnetic parameters of the ferromagnetic core. This influence can appear at frequencies of the core mechanical resonance and lead to changes in the core inductance and quality factor. The effect is relatively weakly pronounced at laminated steel core, but in some cases needs to be considered, e.g. if big size core operates at network frequency. Possibly the magnetostriction can effect on ferrite cores with high-quality mechanical resonant properties. In practical devices it is masked by electric resonances [23], but can reduce core efficiency.

The results of the work were obtained using computational resources of Peter the Great SaintPetersburg Polytechnic University Supercomputing Center (www.scc.spbstu.ru).

\section{References}

1. V. K. Vanin, T. D. Ambrosovskaya, M. G. Popov and S. O. Popov, Power Technology and Engineering, Vol. 50 (1), 87-92 (2016).

2. A.A. Ragozin, M.G. Popov, Electrical Technology Russia, (1) (2002).

3. E. Kharlamova, L. Sakhno, O. Sakhno, International Journal of Applied Engineering Research, Vo. 11, pp. 11055-11059 (2016).

4. E.S. Gorkunov, S.M. Zadvorkin, R. Dimitrov, Kontrol', Diagnostika, No. 1, pp. 12-15 (2017).

5. E.S. Gorkunov, S.M. Zadvorkin, E.A. Putilova, A.A. Bakunova, Inorganic Materials, Vol. 52(15), 1509-1514 (2016).

6. E.S. Gorkunov, S.M. Zadvorkin, E.A. Putilova, Russ. J. Nondestruct.+, 48(8), 495-504 (2012).

7. V.N. Kostin, O.N. Vasilenko, A.M. Porseva and A.A. Kabakova. 10 International Conference on Mechanics, Resource and Diagnostics of Materials and Structures, MRDMS 2016, Ekaterinburg; Russian Federation, AIP Conf. Proc., Vol. 1785, 040029 (2016).

8. D.C. Jiles, D.L. Atherton, J. Magn. Magn. Mater., Vol. 61(1), 48-60 (1986).

9. E.S. Gorkunov, Yu.V. Subachev, A.M. Povolotskaya, S.M. Zadvorkin, Russian Journal of Nondestructive Testing, Vol. 51(9), pp. 563-572 (2015).

10. Stan Zurek, Przeglad Elektrotechniczny. Przeglad Elektrotechniczny, Vol. 2017(7), 1621 (2017).

11. V.N. Kostin, O.N. Vasilenko, D.Yu. Filatenkov, et al, Russ. J. Nondestruct.+, 51(10), 624-632 (2015).

12. E. Cardelli, E. Della Torre and A. Faba, IEEE Trans. Magn., Vol. 45(11), 5243-5246 (2009).

13. J. V. Leite, P. A. da Silva Jr., N. Sadowski, N. Batistela, P. Kuo Peng, and J. P. A. Bastos, IEEE Trans. Magn., Vol. 44(6), 906-909 (2008).

14. Yu. E. Adamian, S. I. Krivosheev, Instrum. Exp. Tech+, № 4, 101-106 (2015).

15. E. Villari, Annu. Rev. Phys. Chem. 126, 87 (1865).

16. Yu. N. Dragoshanskii and V. I. Pudov, Doklady Physics, Vol. 63(9), 358-361 (2018). 
17. Y. Ivanova. 13th National Congress on Theoretical and Applied Mechanics, NCTAM 2017; Institute of Mechanics - Bulgarian Academy of Sciences, Sofia, Bulgaria, MATEC Web of Conferences, Vol. 145, 0500713 (2018).

18. S.V. Bonsovsckiy, Ya.S. Shur, Ferromagnetizm (in Russian). M.,L.: GITTL, 816 p, (1948).

19. C.F. Foo, D.M. Zhang. PESC 98 Record. 29th Annual IEEE Power Electronics Specialists Conference (Cat. No.98CH36196), 2, 1997-2002 (1998).

20. Lixun Zhu, Hee-Sung Yoon, Hyun-Jin Cho, Doo-Jong Um, Chang-Seop Koh, IEEE Trans. Magn., 52(3) (2016).

21. K. Delaere, W. Heylen, R. Belmans, and K. Hameyer, IEEE Trans. Magn., 38(3), 969972, (2002).

22. L. Vandevelde, J.A.A. Melkebeek, IEEE Trans. Magn., 39(3), 1618-1621 (2003).

23. E.J. Meurer, S.W.H. de Haan. 2007 European Conference on Power Electronics and Applications, 1-6 (2007). 